\title{
The Application of Mathematical Morphology Filter in Voltage Flicker Detection
}

\author{
Rui Zhang ${ }^{1,3}$, Yongyang-Zhou ${ }^{1}$ and Huang Jun ${ }^{2,3}$ \\ ${ }^{1}$ School of Automation, Harbin University of Science and Technology, Harbin, \\ China 150080 \\ ${ }^{2}$ Faculty of Geosciences and Environmental Engineering, Southwest Jiaotong \\ University, Chengdu, Sichuan, China 610031 \\ ${ }^{3}$ Chengdu Donglu Traffic Science and Technology Co., Ltd. Chengdu, Sichuan, \\ China 610031 \\ zr_gh@sina.com
}

\begin{abstract}
The analysis of the voltage fluctuation and flicker is important to inhibit flicker. Mathematical morphology filter testing methods are proposed in this paper on the basis of Hilbert transform. Using Hilbert transform to extract flicker envelope, and the mathematical morphological filtering method is used to filter out high frequency noise of the grid. Simulation results show that this method can realize flicker signal detection, determine spectrum of flicker signal through mathematical morphology filtering method, and the measurement precision compared with spectrum amplitude of the signal with noise have larger increase.
\end{abstract}

Keywords: voltage flicker; mathematical morphology filter; Hilbert transform

\section{Introduction}

In recent years, with the continuous application of high power load, the voltage fluctuation and flicker problems for power system is becoming more and more important. Voltage fluctuation and flicker can cause a flicker of light, visual fatigue, motor speed instability and accelerate the aging process. In addition to this, voltage flicker can disrupt accurate electrical equipment and computer system, which can lead to great economic loss. Therefore, it is necessary to find a reliable way to detect voltage fluctuation and flicker signal in electric network to inhibit flicker pollution.

The voltage fluctuation and flicker is a steady-state power quality, the research to the voltage fluctuation and flicker is usually based on amplitude modulation wave frequency modulation of the carrier as the model and the research object. For multiple frequency voltage fluctuation and flicker using wavelet transform to analyze it [1-2]. Due to the change of voltage flicker signal frequency is low, producing flicker signals of harmonic component is usually between the power frequency voltage frequency $50 \mathrm{~Hz}$ or near the frequency doubling harmonic component, it put forward higher requirements to the flicker signal sampling frequency and the selection of wavelet decomposition scale, otherwise it may cause the modulation frequency mixing phenomenon, especially for containing multiple frequency flicker signal wavelet transform directly. Still amplitude or modulated amplitude modulation signal can be separated out, rather than the setting flicker signal .

In this paper, combining the Hilbert transform and mathematical morphology filtering to test the voltage flicker signal, using the mathematical morphological filtering suppresses the higher harmonic components, using the Hilbert transform extract the voltage fluctuation and flicker signal envelope, the detection accuracy compared with the before denoising signal is improved. 


\section{Mathematical Model of Voltage Flicker}

Generally speaking, voltage flicker is regarded as a kind of power frequency voltage signal which modulated by the low frequency fluctuation, after sampling the voltage flicker signals can be represented as model, [3] .

$$
\begin{aligned}
& f(n)=K[1+M(n)] \sin \left(2 \pi n f_{0} / f_{s}+\varphi_{0}\right) \\
& M(n)=\sum_{k=1}^{m} A_{k} \sin \left(2 \pi n f_{k} / f_{s}+\varphi_{k}\right)
\end{aligned}
$$

where $K, f_{0}, \varphi_{0}$ represent the amplitude of the carrier power frequency voltage, frequency and initial Angle $A_{k}, f_{k}, \varphi_{k}$ represent the amplitude of the kind of the $k$ th flicker signal, frequency and initial angle; $M(n)$ is the amplitude of flicker signal. If $U(n)=1+M(n)$, formula (1) can write as follow

$$
f(n)=U(n) \sin \left(2 \pi n f_{0} / f_{s}+\phi_{0}\right)
$$

\section{The Basic Principle of Mathematical Morphology Filtering}

Mathematical morphology [4-5] uses geometry to describe the target signal. The useful information is extracted by the use of structural elements for the characteristics of signal analysis and description.

\subsection{Gray Value Corrosion and Expansion}

Application of mathematical morphology in the signal processing is mainly gray values morphology, the two basic forms are the gray value expansion and gray value corrosion. It can suppose to be processed signal $f(n)$ is a signal sampling to get more than one dimension values, its domain is $D_{f}=\{0,1,2, \ldots N\} ; g(n)$ is a one-dimensional sequence structure elements, its domain is $D_{a}=\{0,1,2, \ldots P\}$; The $P$ and $N$ are integer, $N>P$. The gray value and corrosion are respectively defined as

$$
\begin{gathered}
(f \oplus g)(n)=\max \left\{f(n-m)+g(m) \mid(n-m) \in D_{f}, m \in D_{a}\right\} \\
(f \Theta g)(n)=\min \left\{f(n+m)-g(m) \mid n-m \in D_{f}, m \in D_{a}\right\}
\end{gathered}
$$

where $\oplus$ represents expansion operation; $\Theta$ represents corrosion and trap. Two formula point by point calculation can be understood as: the origin of the structural elements of the translation to the signal on the point of A coincidence, each point of signal combined with every bit of structural elements corresponding (inflation) or subtraction (corrosion) to get the result of he maximum (inflation), or the minimum (corrosion) value. The expansion and corrosion of the point $\mathrm{A}$ are equal to the maximum and minimum values. Inflation is an expansion process which will be cut off wave; Corrosion is a contractive transformation which will fill the trough.

\subsection{Gray Value Open and Close Operation}

Gray value corrosion and expansion is an irreversible operation, the corrosion before expansion is called gray value opened operation, corrosion after inflation called gray value closed operation.

Sequence $f(n)$ of $g(n)$ is defined as opened operation and closed operation respectively,

$$
f \circ g=f \Theta g \oplus g
$$




$$
f \bullet g=f \oplus g \Theta g
$$

Opened operation is commonly used in the actuality to eliminate structural elements with smaller size compared with light details (peak); Commonly used closed operation to eliminate smaller size of structure elements compared dark details (valley).

\subsection{Mathematical Morphology Filtering Algorithm}

The opened and closed operations of mathematical morphology has low-pass characteristic, opened function can be used to filter noise signal at the top of the peak, remove burrs and bridge structure; And closed operation can be used to smooth or inhibit noise signal at the bottom of the troughs, fill a small groove structure. With the cascade form, opened and closed operation can construct various complex morphological filter, filtering effect is better, in order to achieve the effect of the bilateral filter. Based on morphological opened and closed operation can build three kinds of filter, there are alternate filter, alternately hybrid filter, alternating hybrid filter. After opened and closed operation and alternate filter the signal has a clear dc bias, obviously after hybrid filter and alternate mixed filter the signal doesn't have apparent dc bias. This article will select alternate hybrid filter as the mathematical morphology filter, expression as shown in formula (8).

$$
[(f) \operatorname{altmix}(g)](n)=[(f) O C(g)+(f) C O(g)](n) / 2
$$

\subsection{Structural Elements and Selection Principle}

The role of structural elements in morphological operation is similar to the filter window in general signal processing, the shape and size of morphological operations will directly impact on it. Such as selecting different size or shape will directly influence, and selecting structural elements in different size or shape will produce different results. Structural elements have a straight line, curve (such as 2 times, etc.,), 3 inferior, triangle, circle, other polygon (such as a diamond shaped, hexagonal, etc.,) and their combination. A large number of calculation results show that the different signal processing needs a matching structure elements to be better implemented. The selected structural elements should be generally selected as close as possible to analyze signal characteristics of graphics according to the signal characteristics of the structure elements. According to experience, it usually has some kinds of convexity which have a plenty of structural elements in signal processing, generally using the simple geometric shape are round, triangle, etc., The selected structural elements are as close as possible to analyze signal characteristics of graphics.

Morphological operation depends not only on the way of the transformation, but also the effect of filter and related to the shape and size of structural elements. Structural elements will directly affect the monitoring accuracy and speed of dynamic response, it will influence computing speed at the same time. The more complex structure element shape, the bigger the length, the greater the amount of calculation, the opposite is smaller. When the interference suppresses, the more complex shape of structural elements, the greater the interference, but the more time-consuming, the smaller conversely. Usually, filtering white noise uses the semicircle structure, radius should be between 1/50 1/10 signal amplitude, the smaller the radius, the filtering precision is higher; In order to ensure the calculation speed, namely structural elements should be far less than filtering function, the radius of the structural elements should not be bigger than $1 / 4$ of the sampling points per cycle. For the triangular structure elements, if the maximum width of impulse noise is $\mathrm{T}$, on the theory of sampling period for $T_{s}$ to filter the impulse noise, the length of the structural elements you just need to bigger than $T / T_{s}$. But in the practical signal 
processing, it can be appropriately increased length of structural elements in order to obtain better filtering effect.

\subsection{Structure of the Multi-Scale Operator and Error of Sexual Function}

By introducing a variable factor in the process of filtering, it can adjust the width of the structural elements and change the length of the structural elements, more subtle interference in signal is also have detailed filter effect. The start selecting the structure of the operator of length is $r$, variable factor is $a$, then the structure of the multi-scale operator is $r^{\prime}=2^{a} r$. Here, $2^{a}$ is called Variable factor function. For different waveform to be processed, variable factors of selection of the appropriate function are depending on the situation.

For quantitative evaluation of mathematical morphology filtering effect, here introduce a consistency coefficient coef,

$$
\text { coef }=\frac{1}{N} \sqrt{\sum_{i=1}^{N}\left[f_{0}(n)-f_{1}(n)\right]^{2}}
$$

In the type, $N$ is the number of sampling data in perior; $f_{0}(n)$ is the original signal; $f_{1}(n)$ is the signal after morphological filtering.

\section{Hilbert Transform}

Hilbert transform is an important kind of algorithm in signal analysis and processing. Narrow-band signal $x(t)$ can get his conjugate signal $\hat{x}(t)$ by Hilbert transform[6].

$$
\begin{aligned}
& H T[x(t)]=\hat{x}(t)=\frac{1}{\pi} \int_{-\infty}^{+\infty} \frac{x(t)}{t-\theta} d \theta \\
& =x(t) * \frac{1}{\pi t}=x(t) * h(t)
\end{aligned}
$$

$H T[\square]$ represents Hilbert transform, $*$ is convolution, $t$ is time, $\theta$ is variable of integration. The analysis signal $z(t)$ can be represented in formula (10):

$$
z(t)=x(t)=j \hat{x}(t)
$$

the result of the Discrete Fourier transform of formula (10):

$$
\begin{gathered}
Z(k)=X(k)=j X(k)=X(k)+j X(k) H(k) \\
z(k)= \begin{cases}X(k) & k=0 \\
2 X(k) & k=1,2, \ldots \ldots \frac{N}{2}-1 \\
0 & k=\frac{N}{2}, \ldots \ldots . . N-1\end{cases}
\end{gathered}
$$

$z(k)$ is Fourier transform form $z(n), H(k)$ is Fourier transform to Hilbert filter $h(n)$; We can get analysis signal $z(n)$ by doing discrete Fourier inverse transformation to $z(n)$. Doing point by point complex modulus on $\mathrm{A}$, and the calculated sequence of mold is the envelope of the original signal, namely it is flicker signal we are eager to obtain.

\section{Voltage Flicker Signal Detection}


According to the above results, the export signal detection of voltage flicker operation steps are as follows:

Step1: during a measurement cycle, sample voltage changes in an appropriate ratio, and there are a series of voltage sampling values $u(n)(n=0,1,2, \ldots, N-1, N$ is the number of samples ).

Step2: doing discrete Fourier transform to $u(n)$ and the result is $u(k)$.

Step3: using type (11), we can calculate the Fourier transform $Z(k)$ of the analysis signal $z(n)$.

Step4: we can get analysis signal $z(n)$ of $u(n)$ by doing Fourier transform to $u(n)$.

Step5: calculating the model of sequence $z(n)$.

$|z(n)|=\sqrt{u^{2}(n)-\hat{u}^{2}(n)}$

Step6: through mathematical morphology filter filters out harmonic and noise interference, and high amplitude modulated signal can be obtained.

\section{Simulation}

Parameters selection for multiple frequency voltage flicker signal are $A_{1}=1.64 m V, f_{1}=6 \mathrm{~Hz}, A_{2}=1.28 \mathrm{mV}, f_{2}=8 \mathrm{~Hz}$. The sampling frequency is $1600 \mathrm{~Hz}$, sampling time about $4 \mathrm{~s}$, flicker signal containing the signal-to-noise ratio of $20 \mathrm{~dB}$ Gaussian white noise, flicker envelope signal waveform is shown in Figure 1, the corresponding spectrum is shown in Figure 2,

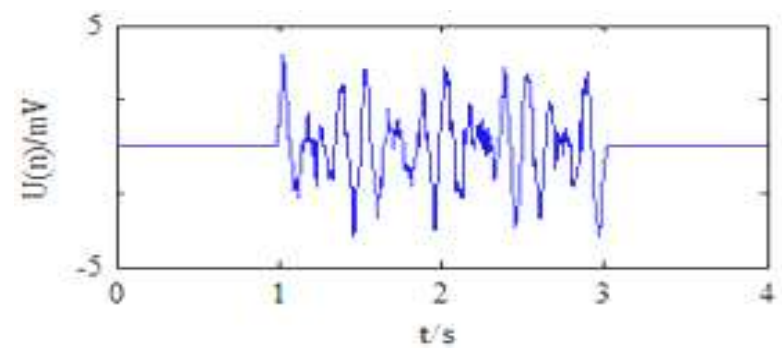

Figure 1. Flicker Envelope with Noise

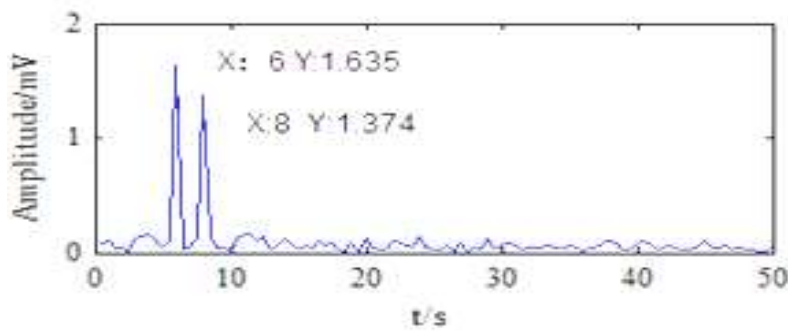

Figure 2. Envelope Spectrum

Select alternate hybrid filter for filtering to sine signal of the positive half cycle for structural elements, selecting structural element radius about 0.01 , the structure of the 
multi-scale operator $r^{\prime}=2^{a} r$. coef almost attained minimum value when $a=-4$. After mathematical morphology filter filtering, flicker signal envelope is shown in Figure 3, the corresponding spectrum is shown in Figure 4. The effect of Denoise is used to determine the noise spectrum of the measured relative error of flicker signals before and after, the results are shown in Table 1.

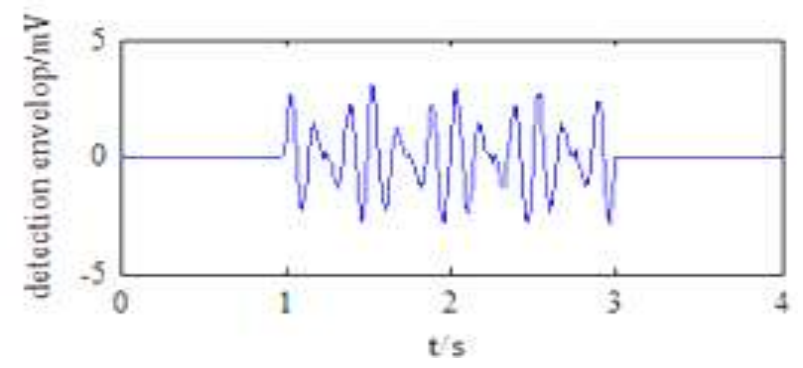

Figure 3. The Envelope of Flicker after Filtering

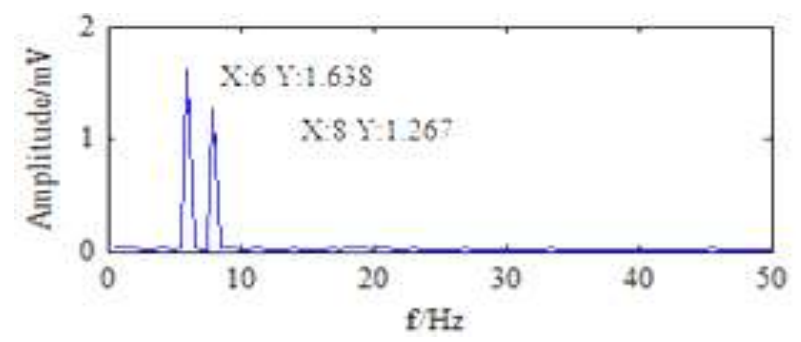

Figure 4. Envelope of Signal Spectrum

Table 1. Comparing Results for Square Flicker Signal Detection

\begin{tabular}{l|c|c|c}
\hline & True value & Flick with noise & Removed noise \\
\hline Frequency/Hz & 6 & $0 \%$ & $0 \%$ \\
\hline Amplitude/mV & 1.64 & $0.781 \%$ & $0.122 \%$ \\
\hline Frequency/Hz & 8 & $0 \%$ & $0 \%$ \\
\hline Amplitude/mV & 1.28 & $7.343 \%$ & $1.015 \%$ \\
\hline
\end{tabular}

\section{Conclusion}

This paper present a new method based on Hilbert transform and mathematical morphology filtering for voltage flicker detection, the method using the Hilbert transform realize the envelope extraction of the voltage fluctuation and flicker signal. Using mathematical morphology filter, and the method for higher harmonic part of the power grid has good inhibition, the effectiveness of the proposed algorithm is verified by simulation experiments.

\section{Acknowledgements}

This work was supported by Applied Fundamental Research Plan Project of Sichuan Province of China (No.2015JY0234).

\section{References}

[1] R. Zhang, G. Q.Liu, L.Y. Zhang and X.H. Zhang, "The detection of voltage flicker based on complex wavelets", 3rd international Conference on Measuring Technology and 
Mechatronics Automation, vol. 48, (2011), pp. 1231-1234.

[2] X. L. Wen and T. Chen, "Measurement of voltage fluctuation and flicker in electric power system based on wavelet transform", International Conference on Wavelet Analysis and Pattern Recognition, (2007), pp. 1822-1826.

[3] H. X. Zhao, M. Z. Chen and H. Z. Dai, "Error analysis of discrete calculation method of flicker severity and application", Power system technology, vol. 28, no. 13, (2004), pp. 84-87.

[4] J. Serra, "Morphological Filtering:An Overview", Signal Processing, vol. 38, no. 4, (1994), pp. 3-11.

[5] F. H. Xing, M. Yan, L. Yi and N. Gui, "Detection of transient power quality disturbance based on morphology", Electrical Measurement\&Instrumentation, vol. 52, no. 17, (2015).

[6] D. Yi, X. Wei, D. Yang and M. Li, "Demodulation of non-stationary amplitude modulated signal based on Hilbert transform", Journal of vibration and shock, vol. 32, no. 10, (2013). 
International Journal of u- and e- Service, Science and Technology

Vol. 10, No. 1 (2017) 